\title{
Oblique Scattering from Lossy Periodic Surfaces with Application to Anechoic Chamber Absorbers
}

\author{
Ramakrishna Janaswamy, Member, IEEE
}

\begin{abstract}
Plane wave scattering from lossy, periodic surfaces with periodicity in one direction is considered for arbitrary polarization and incidence angles. The material is assumed to be homogeneous, characterized by complex values of permittivity and permeability. Müller type coupled integral equations are derived for the surface electric and magnetic currents. Power reflection coefficient is defined in terms of the scattered far fields. The integral equations are solved by the boundary element method (BEM) with constant elements. Arbitrary surfaces are accomodated by approximating the actual profile by means of linear segments. Other speed-up techniques are utilized to generate a numerically efficient solution. Substantial comparison is made for special cases to verify the formulation. Reflection coefficient is calculated for a variety of surface shapes such as rectangular, sinusoidal and triangular. A new profile shape is presented that results in a lower reflection coefficient compared to the commonly used triangular shape. For the specific case of the triangular profile, a systematic study is carried out for the dependence of reflection coefficient on various parameters.
\end{abstract}

\section{INTRODUCTION}

$\mathrm{A}^{\mathrm{N}}$ $\mathrm{N}$ anechoic chamber is an important segment in the characterization of antennas and scatterers. The walls of an anechoic chamber are lined with lossy (absorbing) material to simulate a near-free space environment. The front surface of the absorber is often shaped in the form of an array of wedges or pyramids to reduce reflections from the air-absorber interface. This will also result in broad-banding. A brief history and survey of work done in the areas of absorbers and anechoic chambers prior to 1973 is discussed in [1]. Most of the earlier work appears to be experimental in nature although some theoretical work has been reported in the past. Bucci and Franceschetti [2] studied scattering from wedge type absorbers at normal incidence for the two limiting cases of low and high frequencies. Recently, DeWitt and Burnside [3] studied scattering from pyramidal and wedge shaped absorbers using the geometric theory of diffraction (GTD). However, the results are only applicable to the high frequency case where the dimensions of the pyramids/wedges are at least several times larger than the wavelength of the

Manuscript received June 10, 1991; revised October 31, 1991. This work was supported by the Naval Weapons Center, China Lake, CA and the Naval Postgraduate School, Monterey, CA.

The author is with the Department of Electrical and Computer Engineering, Naval Postgraduate School, Code EC/Js, Monterey, CA 93943.

IEEE Log Number 9105958 incident wave. Additional data similar to those presented in [3] are given in [4]. Cheon and Liepa [5] performed a full wave analysis and reported results on oblique wave scattering for the special case of wedge type absorbers. A great deal of work has been carried out by the optics community for scattering by singly periodic conductive and dielectric surfaces under the heading gratings [6]. In the majority of the works, the incident wave is assumed to lie in the plane normal to the generators of the periodic surface and the results are of limited use to the absorber designer. In an actual chamber the waves are incident from all angles of incidence and the amount of power scattered by the absorber surface depends on a number of factors including the frequency, polarization and aspect angles of the incident wave, the material constitutive parameters, the surface profile parameters, and the thickness of the absorber panel.

In this work, a systematic approach is undertaken to study oblique wave scattering by arbitrarily shaped, one-dimensional, periodic, lossy surfaces. Side walls, floor, and ceiling of rectangular as well as tapered chambers make use of such surfaces. Several profile shapes such as the rectangular pulse, sinusoidal, triangular, and a variant of triangular are considered. The incident plane wave is allowed to have any polarization and aspect angles. In the analysis, the thickness of the absorber is assumed to be infinitely large which is a reasonable approximation for a good absorber. Coupled integral equations are derived involving the surface currents which are then solved by the boundary element method. Effects of the profile geometry, material constitutive parameters, polarization, frequency and aspect angles of the incident wave on the power scattered by the surface are studied.

In Section II, the required integral equations are presented. A power reflection coefficient is defined in terms of the scattered far fields. A numerical procedure is presented involving the boundary element method (BEM). In Section III, numerical results are presented. Finally, in Section IV, a summary of the results is presented.

\section{THEORY}

Fig. 1 shows the geometry of the problem. A uniform plane wave is incident from medium 1 having constitutive parameters $\left(\epsilon_{e} \epsilon_{0}, \mu_{e} \mu_{0}\right)$ onto a lossy half-space (medium 2) bounded by the surface $S$. The permittivity and permeability, respectively, of vacuum are $\epsilon_{0}$ and $\mu_{0}$. Medium 2 is characterized by a complex permittivity $\epsilon_{0} \epsilon_{r}$ and complex perme- 


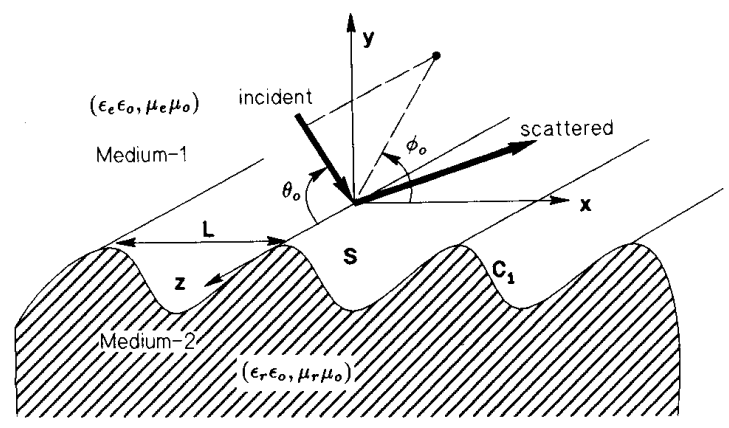

Fig. 1. Plane wave incident on a periodic lossy surface. The incident wave is characterized by spherical angles $\theta_{0}$ and $\phi_{0}$ and is of arbitrary polarization.

ability $\mu_{0} \mu_{r}$. The surface $S$ is periodic in the $x$-direction with a period $L$ and is uniform in the $z$-direction. The corrugations $C_{1}$ corresponding to one period of the surface are defined by $y=f(x)$. An $e^{j \omega t}$ time dependence is assumed for all fields and is suppressed throughout.

\section{A. Development of Coupled Integral Equations}

Several integral equation formulations are available for studying electromagnetic scattering by homogeneous, penetrable bodies [7], [8]. We have chosen the formulation based on the Müller type integral equations [9]. Use of these integral equations facilitates employment of simpler basis functions, such as pulse functions, in the numerical solution, thus, resulting in an efficient, general purpose procedure for handling a wide variety of surface profile shapes.

Although the incident field can be decomposed into a transverse magnetic, $\operatorname{TM}_{z}\left(H_{z}=0\right)$, and a transverse electric, $\mathrm{TE}_{z}\left(E_{z}=0\right)$ polarizations, the boundary conditions prevent a similar decomposition for the total field for the general case of oblique incidence [10]. We shall use TM/TE decomposition for the incident field alone. The superscript $z$ will be dropped hereupon for simplicity of notation. Note that the meanings attached here to TE/TM decomposition are different from those of [11] and the rest of the optics community [6].

The incident electric field $\vec{E}^{i}$ can be expressed as

$$
\begin{aligned}
\vec{E}^{i} & =-\eta_{1} H_{0} \hat{\theta} e^{j\left(k_{x} x+k_{y} y+k_{z} z\right)}, \\
& =E_{0} \hat{\phi} e^{j\left(k_{x} x+k_{y} y+k_{z} z\right)},
\end{aligned}
$$

where $k_{x}=k_{1} \sin \theta_{0} \cos \phi_{0}, k_{y}=k_{1} \sin \theta_{0} \sin \phi_{0}$ and $k_{z}$ $=k_{1} \cos \theta_{0}$. The wavenumber $k_{1}$ and intrinsic impedance $\eta_{1}$ in medium 1 are $k_{1}=k_{0} \sqrt{\epsilon_{e} \mu_{e}}, \eta_{1}=\eta_{0} \sqrt{\mu_{e} / \epsilon_{e}}, k_{0}$ and $\eta_{0}$ being similar quantities for free space. The radian frequency of the incident wave will be denoted by $\omega$. The angles $\theta_{0}$ and $\phi_{0}$ are the usual spherical coordinates as defined in Fig. 1 and $\hat{\theta}$ and $\hat{\phi}$ are the usual unit vectors in the spherical coordinate system. The constants $E_{0}$ and $H_{0}$ signify the relative amplitudes of TE and TM components, respectively.

The starting point for the formulation of the integral equations is the set given by Müller $[9$, p. 301]. Denoting the unit normal on the surface $S$ as $\hat{n}$ and the surface electric and magnetic currents as $\overrightarrow{\mathscr{J}}=\hat{n} \times \vec{H}$ and $\overrightarrow{\mathscr{M}}=-\hat{n} \times \vec{E}$, respectively, we have

$$
\begin{aligned}
& \frac{\overrightarrow{\mathscr{M}}}{2}=\frac{\epsilon_{e}}{\epsilon_{e}+\epsilon_{r}} \overrightarrow{\mathscr{M}}^{i}+\frac{\hat{n} \times}{4 \pi\left(\epsilon_{e}+\epsilon_{r}\right)} \iint_{S} \overrightarrow{\mathscr{M}} \\
& \times \nabla^{\prime}\left(\epsilon_{e} \varphi_{1}-\epsilon_{r} \varphi_{2}\right) d s^{\prime} \\
& +\frac{j \eta_{0} \hat{n} \times}{4 \pi k_{0}\left(\epsilon_{e}+\epsilon_{r}\right)} \iint_{S}\left[\overrightarrow{\mathscr{J}}\left(k_{1}^{2} \varphi_{1}-k_{2}^{2} \varphi_{2}\right)\right. \\
& \left.+\left(\overrightarrow{\mathscr{J}} \cdot \nabla^{\prime}\right) \nabla^{\prime}\left(\varphi_{1}-\varphi_{2}\right)\right] d s^{\prime} \\
& \frac{\overrightarrow{\mathscr{J}}}{2}=\frac{\mu_{e}}{\mu_{e}+\mu_{r}} \overrightarrow{\mathscr{J}}^{i}+\frac{\hat{n} \times}{4 \pi\left(\mu_{e}+\mu_{r}\right)} \iint_{S} \overrightarrow{\mathscr{J}} \\
& \times \nabla^{\prime}\left(\mu_{e} \varphi_{1}-\mu_{r} \varphi_{2}\right) d s^{\prime} \\
& -\frac{j \hat{n} \times}{4 \pi k_{0} \eta_{0}\left(\mu_{e}+\mu_{r}\right)} \iint_{S}\left[\overrightarrow{\mathscr{M}}\left(k_{1}^{2} \varphi_{1}-k_{2}^{2} \varphi_{2}\right)\right. \\
& \left.+\left(\overrightarrow{\mathscr{M}} \cdot \nabla^{\prime}\right) \nabla^{\prime}\left(\varphi_{1}-\varphi_{2}\right)\right] d s^{\prime}
\end{aligned}
$$

where $\varphi_{p}=e^{-j k_{p} r} / r, p=1,2$, are the Green's functions in the two regions, $k_{2}=k_{0} \sqrt{\mu_{r} \epsilon_{r}}$, and a prime denotes quantities defined with respect to the source coordinates. Note that the equations given in [7] and [9] contain algebraic errors. The correct forms are as given above. Equations (3) satisfy the principle of duality, whereas the ones given in [7] and [9] do not. The above integrals are understood to be of the Cauchy's principal value type. Two points are worth noting concerning (3). Firstly, the integral equations do not involve any derivatives of the surface currents. This is an important advantage from a numerical standpoint, for it will permit discontinuous representation of current such as a staircase approximation resulting from the use of pulse basis functions. Secondly, although higher order derivatives operate on the Green's functions, they will not increase the order of the singularity of the kernels because they operate on the combined function $\left(\varphi_{1}-\varphi_{2}\right)$ which is less singular than either $\varphi_{1}$ or $\varphi_{2}$. This point will become more clear shortly.

We now make use of the one dimensional nature and periodicity of the surface $S$. Since the structure is uniform in the $z$ direction, all fields and currents will have the same behavior, $e^{j k_{z} z}$, in the $z$ direction as the incident field. Combining this with the Floquet's theorem for a periodic structure, we write

$$
\begin{gathered}
\overrightarrow{\mathscr{M}}\left(x^{\prime}+m L, y^{\prime}, z^{\prime}\right)=\vec{M}\left(x^{\prime}, y^{\prime}\right) e^{j\left(k_{x} m L+k_{z} z\right)}, \\
m=-\infty, \cdots, \infty \\
\overrightarrow{\mathscr{J}}\left(x^{\prime}+m L, y^{\prime}, z^{\prime}\right)=\vec{J}\left(x^{\prime}, y^{\prime}\right) e^{j\left(k_{x} m L+k_{z} z\right)}, \\
m=-\infty, \cdots, \infty .
\end{gathered}
$$

Equations (4) and (5) are substituted into (3) and the integral equations are enforced in the $z=0$ plane. Integration with respect to $z$ can be performed in a closed form and the integral over the corrugations of the surface can be converted to one over a single period $C_{1}$. The electric and magnetic surface currents are resolved into components along 
the $z$ axis and along the unit tangent vector $\hat{\tau}$ to the curve $C_{1}$ as $\vec{J}=\hat{\tau} J_{1}+\hat{z} J_{2}$, and $\vec{M}=\hat{\tau} M_{1}+\hat{z} M_{2}$ to finally result in a set of four scalar equations for the four unknowns. The final equations are

$$
\frac{M_{1}}{2}=\frac{\epsilon_{\mathrm{e}} M_{1}^{i}}{\left(\epsilon_{e}+\epsilon_{r}\right)}+\int_{C_{1}}\left[G_{11} M_{1}+G_{13} J_{1}+G_{14} J_{2}\right] d l^{\prime}
$$

$$
\begin{aligned}
\frac{M_{2}}{2} & =\frac{\epsilon_{e} M_{2}^{i}}{\left(\epsilon_{e}+\epsilon_{r}\right)} \\
& +\int_{C_{1}}\left[G_{21} M_{1}+G_{22} M_{2}+G_{23} J_{1}+G_{24} J_{2}\right] d l^{\prime}
\end{aligned}
$$$$
\frac{J_{1}}{2}=\frac{\mu_{e} J_{1}^{i}}{\left(\mu_{e}+\mu_{r}\right)}+\int_{C_{1}}\left[G_{31} M_{1}+G_{32} M_{2}+G_{33} J_{1}\right] d l^{\prime}
$$

$$
\begin{aligned}
\frac{J_{2}}{2} & =\frac{\mu_{e} J_{2}^{i}}{\left(\mu_{e}+\mu_{r}\right)} \\
& +\int_{C_{1}}\left[G_{41} M_{1}+G_{42} M_{2}+G_{43} J_{1}+G_{44} J_{2}\right] d l^{\prime}
\end{aligned}
$$

The various kernel functions $G_{p q}, p=1, \cdots, q=1, \cdots$ are

$$
\begin{gathered}
G_{11}=\frac{-j \frac{\partial}{\partial n^{\prime}}\left(\epsilon_{e} \psi_{1}-\epsilon_{r} \psi_{2}\right)}{4\left(\epsilon_{e}+\epsilon_{r}\right)} \\
G_{13}=\frac{-j k_{z} \eta_{0} \frac{\partial}{\partial l^{\prime}}\left(\psi_{1}-\psi_{2}\right)}{4 k_{0}\left(\epsilon_{e}+\epsilon_{r}\right)} \\
G_{14}=\frac{\eta_{0}\left(k_{\rho 1}^{2} \psi_{1}-k_{\rho 2}^{2} \psi_{2}\right)}{4 k_{0}\left(\epsilon_{e}+\epsilon_{r}\right)} \\
G_{21}=\frac{k_{z} \sin \left(\zeta^{\prime}-\zeta\right)\left(\epsilon_{e} \psi_{1}-\epsilon_{r} \psi_{2}\right)}{4\left(\epsilon_{e}+\epsilon_{r}\right)} \\
G_{22}=\frac{-j\left[\sin \left(\zeta^{\prime}-\zeta\right) \frac{\partial}{\partial l^{\prime}}+\cos \left(\zeta^{\prime}-\zeta\right) \frac{\partial}{\partial n^{\prime}}\right]\left(\epsilon_{e} \psi_{1}-\epsilon_{r} \psi_{2}\right)}{4\left(\epsilon_{e}+\epsilon_{r}\right)}
\end{gathered}
$$$$
G_{23}=\frac{-\eta_{0}}{4 k_{0}\left(\epsilon_{e}+\epsilon_{r}\right)}\left[\cos \left(\zeta^{\prime}-\zeta\right)\left(k_{1}^{2} \psi_{1}-k_{2}^{2} \psi_{2}\right)\right.
$$$$
+\left\{\cos \left(\zeta^{\prime}-\zeta\right)\left[\frac{\partial^{2}}{\partial l^{\prime 2}}-\kappa \frac{\partial}{\partial n^{\prime}}\right]\right.
$$$$
\left.\left.-\sin \left(\zeta^{\prime}-\zeta\right)\left[\kappa \frac{\partial}{\partial l^{\prime}}+\frac{\partial^{2}}{\partial l^{\prime} \partial n^{\prime}}\right]\right\}\left(\psi_{1}-\psi_{2}\right)\right]
$$$$
G_{24}=-\frac{-j k_{z} \eta_{0} \sin \left(\zeta^{\prime}-\zeta\right)}{4 k_{0}\left(\epsilon_{e}+\epsilon_{r}\right)} \frac{\partial}{\partial n^{\prime}}\left(\psi_{1}-\psi_{2}\right)
$$$$
-\cos \left(\zeta^{\prime}-\zeta\right) G_{13}
$$

In the preceding equations, $l^{\prime}$ stands for the arc length parameter and $\partial / \partial n^{\prime}$ denotes normal derivative on $C_{1}$ at the source point. The angles which the unit tangents at the source and the observation points make with the $x$-axis are denoted by $\zeta^{\prime}$ and $\zeta$, respectively, and $\kappa$ represents the curvature of $C_{1}$ at the source point. The quantity $\psi_{p}$ is the plane-wave spectrum representation of the periodic Green's function, which is obtained by the application of Poisson's sum formula to the Hankel function representation [11], [12] and is given by

$\psi_{p}=\frac{2}{L} \sum_{m=-\infty}^{\infty} \frac{e^{j\left(2 m \pi / L+k_{x}\right)\left(x-x^{\prime}\right)-j K_{m p}\left|y-y^{\prime}\right|}}{K_{m p}}$,

$$
p=1,2
$$

where

$$
\begin{aligned}
K_{m p} & =\sqrt{k_{\rho p}^{2}-\left(2 m \pi / L+k_{x}\right)^{2}} \\
& =-j \sqrt{\left(2 m \pi / L+k_{x}\right)^{2}-k_{\rho p}^{2}},
\end{aligned}
$$

and

$$
k_{\rho p}^{2}=k_{p}^{2}-k_{z}^{2} .
$$

Equations for the other kernel functions can be obtained from those given above by referring to (6c) and (6d) and applying the principle of duality. For $m \rightarrow \infty, K_{m 2} \rightarrow K_{m 1}$ and the summands in $\psi_{1}$ and $\psi_{2}$ approach each other. Higher order derivatives operating on $\psi_{p}, p=1,2$ tend to slow the numerical evaluation of the resulting summations. However, since they operate on $\left(\psi_{1}-\psi_{2}\right)$, the numerical convergence of the series is not degraded in view of the above fact.

For incidence in the normal plane, $k_{z}=0$, which results in $G_{13}=G_{21}=G_{24}=G_{31}=G_{42}=G_{43}=0$. This causes (6) to decouple into separate TM and TE sets. Equations (6a) and $(6 \mathrm{~d})$ can then be solved for the TM case. Likewise, (6b) and $(6 c)$ can be solved for the TE case.

\section{B. Far-Zone Scattered Fields}

After equations (6a) and (6b) have been solved for the surface currents, the far-zone scattered electric field $\vec{E}^{s}$ in the $z=0$ plane can be obtained from

$$
\begin{aligned}
\vec{E}^{s}=\frac{j}{4} \int_{C_{1}}\left[j k_{1} \eta_{1} \vec{J} \psi_{1}+\vec{M}\right. & \times \nabla^{\prime} \psi_{1} \\
& \left.+j \frac{\eta_{1}}{k_{1}}\left(\vec{J} \cdot \nabla^{\prime}\right) \nabla^{\prime} \psi_{1}\right] d l^{\prime}
\end{aligned}
$$

where the del operator $\nabla^{\prime}$ is written in terms of the transverse $\mathrm{del}, \nabla_{t}$, as $\nabla^{\prime}=\nabla_{t}^{\prime}-j k_{z} \hat{z}$. Using (14), we may express the scattered electric field as

$$
\vec{E}^{s}(x, y)=\sum_{m=-\infty}^{\infty} \vec{E}_{m}^{s} e^{j\left(2 \pi m / L+k_{x}\right) x-j K_{m !} y} .
$$

The scattered field is seen to comprise of an infinite number of plane waves each having its own vector amplitude $\vec{E}_{m}^{s}$ and direction of propagation. For a given period $L$ and frequency $\omega$, only a finite number of these (for which $K_{m 1}$ is real) will 
carry power to the far field, and the rest are evanescent in nature. The angles of departure $\left(\theta_{m}^{s}, \phi_{m}^{s}\right)$ of $m$ th propagating order are

$$
\begin{gathered}
\theta_{m}^{s}=\pi-\theta_{0}, \\
\phi_{m}^{s}=\pi-\cos ^{-1}\left(\cos \phi_{0}+\frac{2 m \pi}{k_{1} L \sin \theta_{0}}\right) .
\end{gathered}
$$

Note that the angles are dependent only on the period $L$, but not on the exact shape of the surface profile. The shape will, however, affect the amplitude $\vec{E}_{m}^{s}$. The directions of the scattered plane waves will all lie on a cone having half-angle $\theta_{0}$ with its axis along the negative $z$ axis. Except for the specularly reflected, zeroth-ordered wave $\left(\phi_{0}^{s}=\pi-\phi_{0}\right)$, all the higher order waves will first graze the surface before entering the upper half-space. That is, when a particular higher order wave is just excited, its scattering angle $\phi_{m}^{s}$ is equal to either 0 or $\pi$. Whether or not a particular order is excited depends on the frequency, angles of incidence, and the period of the profile.

Denoting by $-M_{-}$and $M_{+}$the lowest and the highest propagating orders, respectively (obtainable from (17)), a power reflection coefficient $R$ may be defined as

$$
R=\sum_{m=-M_{-}}^{M_{+}} P_{m}^{r} \triangleq \sum_{m=-M_{-}}^{M_{+}} \frac{\left|E_{m}^{s}\right|^{2} \sin \phi_{m}^{s}}{\left(E_{0}^{2}+\eta_{1}^{2} H_{0}^{2}\right) \sin \phi_{0}}
$$

where $P_{m}^{r}$ is the normalized power carried by the $m$ th propagating order in the positive $y$-direction. For a given frequency, profile period, and incidence angles, a higher order wave just excited (in the sense of the above paragraph) will not affect the reflection coefficient, although it will carry power along the surface. Note that the above definition of reflection coefficient includes the contribution of all orders and not just the specular component ( $m=0$ term) as is done in [2].

\section{Numerical Solution Procedure}

Equations (6a) and 6(b) are solved numerically by the boundary element method [13]. The method has been applied to electromagnetic wave problems before [14]. The basic idea is to discretize the boundary integral equations (6) by applying boundary elements. In terms of the moment method, it is equivalent to using subsectional basis functions and point matching procedure. However, shape functions corresponding to subsectional bases are taken systematically as in the finite element method. This implies that the boundary shape is approximated by a linear segments when pulse or triangular expansion functions are used [13]. This would help enhance the universality of the method in the sense that any complicated shape can automatically be approximated by entering only the coordinates of mesh points of subdivided elements.

Constant elements (pulse expansion functions) and linear segmentation are employed here. An advantage with this choice of elements is that all integrals in (6) can be performed in a closed form in terms of infinite series. No special conditions are imposed at a vertex point in the case of piecewise continuous profiles. The exact nature of singularity of transverse fields near a dielectric wedge is not known yet [18] for it to be implemented into the numerical scheme. However, it is believed that the far fields are nor significantly affected by the singular behavior of the fields near the wedge.

The integral equations are enforced at the midpoint of each element to finally result in a matrix equation for the unknown surface currents. Shank's acceleration scheme [15] for series summations is used to further speed up the matrix fill-time. Finally, standard techniques are used to solve the matrix equation for the unknown current coefficients.

\section{Numerical Results and Discussion}

The boundary shape was approximated by linear segments having a fixed chord length. This will result in a better representation of an arbitrary profile that with uniform sampling along the $x$-axis. The use of Shank's transformation led to significant savings in the computational time. All of the series could be computed to a relative accuracy of $10^{-5}$ using less that 1000 terms. Numerical convergence for the current distribution as well as for the reflection coefficient was obtained with 30 nodes per wavelength in medium 1 along the chord length. We have maintained at least 30 nodes per wavelength in all the results shown here. No special symmetry of the structure was exploited so as to keep the code as general as possible. In all the results presented here, we will assume $\epsilon_{e}=1, \mu_{e}=1, \mu_{r}=1$ and denote the free-space wavelength by $\lambda_{0}$. Furthermore, the designations TM/TE are used to indicate the polarizations of the incident wave.

In order to validate the formulation, we have compared our numerical results to those presented in various references for specific geometries and incidence angles. Comparison was made for current, scattered fields and power. In every case, a very good agreement was found. Tables I and II exemplify the kind of agreement obtained. In the cases of references cited, data shown was taken from plots presented. The slight disagreement for small values of reflected power could be a result of this. The value of $\epsilon_{r}$ in Table II is that of gold at optical frequencies and corresponds to a refractive index value of $0.15-j 4.65$.

To illustrate the coupling between TM and TE modes for oblique incidence, the total surface current induced on a triangular surface for a TM polarized incident wave is shown in Fig. 2. Results are shown for $\phi_{0}=90^{\circ}, \theta_{0}=45^{\circ}, \epsilon_{r}=$ $1.45-j 0.4$ and $H_{0}=1$. The arc length value is zero at the initial base point of the triangle. In the plot shown, the current amplitudes at the match points have been joined to produce a smooth curve. The nonzero amplitudes of $M_{2}$ and $J_{1}$ signify coupling to a TE mode.

Results will now be presented for scattering by typical absorber profile shapes. Most commercially available absorber panels are made from carbon impregnated urethane foam with protective layers of paint and fire retardents. Values of absorber dielectric constant depend upon carbon concentration and vary with frequency among other things. We shall use a value of $\epsilon_{r}=1.45-j 0.4$ specified at $2 \mathrm{GHz}$ as quoted by one of the companies. The skin depth for this material is around $0.97 \lambda_{0}$. All the shapes considered here are 


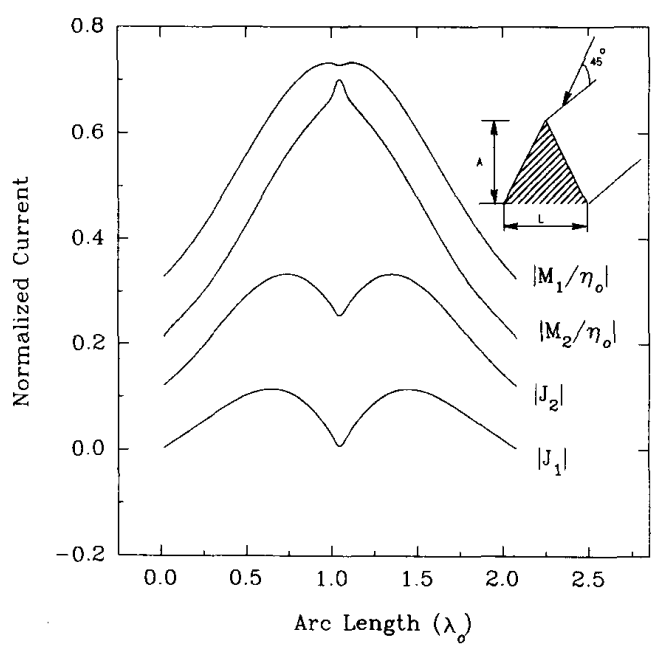

Fig. 2. Normalized magnitude of total surface current for oblique incidence. The parameters of the isosceles triangle are $A=1.016 \lambda_{0}$ and $L=0.508 \lambda_{0}$.

TABLE I

TM Scattering by Rectangular Pulse

\begin{tabular}{ccccc}
\hline$\phi_{0}$ & \multicolumn{2}{c}{ Present } & \multicolumn{2}{c}{ [16] } \\
(Deg.) & $P_{0}^{r}$ & $P_{-1}^{r}$ & $P_{0}^{r}$ & $P_{-1}^{r}$ \\
\hline 20 & 0.378 & $1.88 \times 10^{-2}$ & 0.372 & $2.0 \times 10^{-2}$ \\
30 & 0.238 & $2.28 \times 10^{-2}$ & 0.238 & $2.3 \times 10^{-2}$ \\
40 & 0.162 & $2.26 \times 10^{-2}$ & 0.161 & $2.2 \times 10^{-2}$ \\
50 & 0.135 & - & 0.135 & - \\
60 & 0.106 & - & 0.105 & - \\
70 & 0.056 & - & 0.058 & - \\
\hline
\end{tabular}

$\epsilon_{r}=4, L=0.6 \lambda_{0}=$ pulse height, pulse width $=L / 2, \theta_{0}=90^{\circ}$.

TABLE II

Te Scattering by Fourier Profile

\begin{tabular}{ccccc}
\hline $\begin{array}{c}\phi_{0} \\
\text { (Deg.) }\end{array}$ & $P_{0}^{r}$ & $P_{-1}^{r}$ & $P_{0}^{r}$ & $P_{-1}^{r}$ \\
\hline 10 & 0.928 & 0.050 & 0.929 & 0.058 \\
20 & 0.925 & 0.026 & 0.927 & 0.030 \\
30 & 0.909 & 0.027 & 0.934 & - \\
40 & 0.701 & 0.157 & 0.757 & 0.157 \\
50 & 0.311 & 0.527 & 0.349 & 0.547 \\
60 & 0.113 & 0.730 & 0.116 & 0.788 \\
70 & 0.394 & 0.480 & 0.424 & 0.493 \\
80 & 0.875 & 0.035 & 0.904 & 0.035 \\
\hline
\end{tabular}

$\epsilon_{r}=-21.6-j 1.4, \quad L=\lambda_{0}, \quad f(x)=0.24 \lambda_{0}[\cos (2 \pi x / L)+$ $0.2 \cos (4 \pi x / L)], \theta_{0}=90^{\circ}$

self-complementary in nature. That is, when cut from an absorber block, the shapes of the absorber surface and of the surface left behind are identical. This is an important economical concern to the absorber designer. We shall progress successively from a blunt surface such as a rectangle to a pointed surface such as triangle.

Figs. 3-5 show reflection coefficient as a function of aspect angle for rectangular, sinusoidal and triangular shapes. The heights of corrugations are slightly over one skin depth. Angles shown on the horizontal axis are measured from the $y$-axis for incidence in the two principal planes: $x y\left(\theta_{0}=\right.$

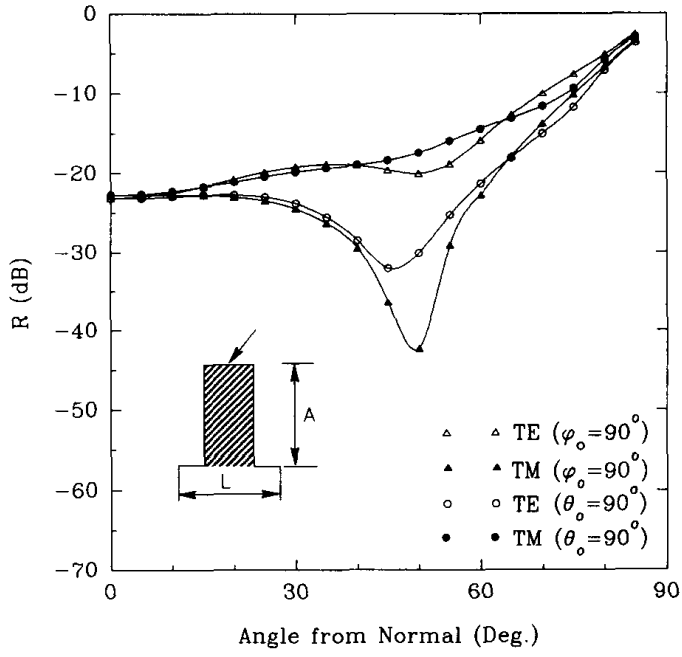

Fig. 3. Variation of reflection coefficient with incidence angle for rectangular profile. The duty cycle of the rectangle is 0.5 with $A / \lambda_{0}=1.016$ $L / \lambda_{0}=0.508$ and $\epsilon_{r}=1.45-j 0.4$

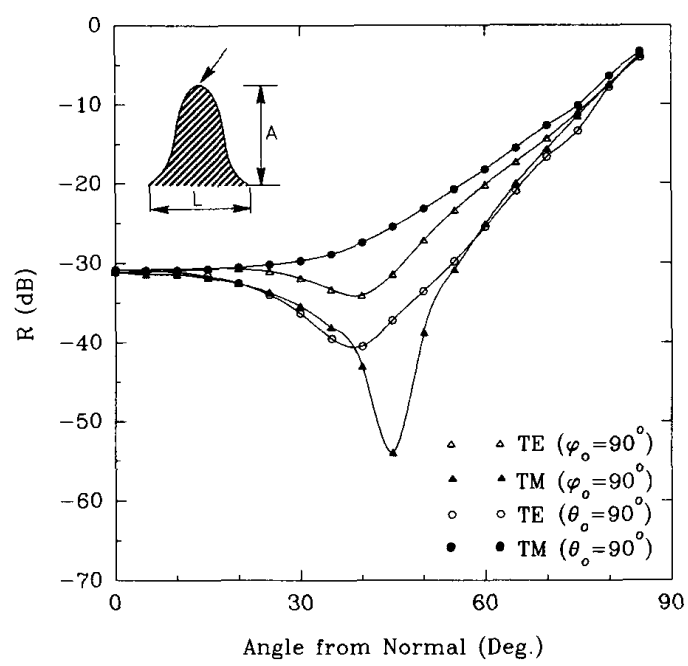

Fig. 4. Variation of reflection coefficient with incidence angle for a sinusoidal profile with $A / \lambda_{0}=1.016, L / \lambda_{0}=0.508$ and $\epsilon_{r}=1.45-j 0.4$.

$\left.90^{\circ}\right)$ and $y z\left(\phi_{0}=90^{\circ}\right)$. The term normal is understood to mean $\theta_{0}=\phi_{0}=90^{\circ}$. The duty cycle of the rectangular pulse is assumed to be 0.5 . For the sake of comparison, the reflection coefficient for a flat surface at normal incidence and having the same constitutive parameters as above is $-18.27 \mathrm{~dB}$. It is seen that while all the three surfaces reflect significantly for angles near grazing, the triangular profile produces the lowest reflection coefficient for angles near normal, followed by the sinusoidal and the rectangular. The amount of power absorbed by a surface depends on the surface shape, the material bulk present in the corrugations in one period, as well as on the constitutive parameters of the absorber material. All the three surfaces considered above have the same material constitutive parameters and approxi- 


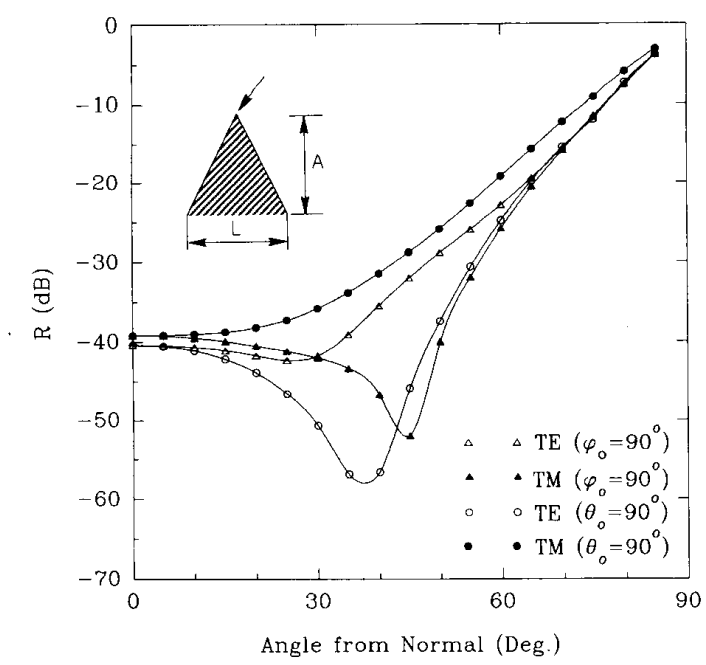

Fig. 5. Variation of reflection coefficient with incidence angle for an isosceles triangular profile with $A / \lambda_{0}=1.016, L / \lambda_{0}=0.508$ and $\epsilon_{r}=$ $1.45-j 0.4$.

mately the same cross-sectional area per period. However, it appears that the triangular profile offers the smoothest transition upon entry from free space to the material half-space, thus, resulting in the lowest reflection coefficient. Far away from the normal, none of the surfaces provides a smooth transition as evidenced by the high reflection coefficient.

It may be tempting to consider more pointed structures than the triangular one in an attempt to further reduce near-normal returns. Preliminary ideas were considered in [4]. However, the return will not be significantly lowered unless there is enough material in the corrugations. To verify this, the sloping sides of the triangle above were replaced with exponential curves such that the profile height decayed more rapidly with distance. Results revealed that it did not perform any better than the triangular one and that the reflections were actually higher compared to the triangle. This is attributed to the fact that the exponential profile contains lesser material in the corrugations than the triangular one. To provide a smoother transition to free space, while at the same time maintaining enough material to absorb, we shall consider a profile that is more pointed than the triangular, but one that is broader at the base to compensate for the loss of material near the vertex. Fig. 6 shows the reflection coefficient versus angle for such a profile. It is seen that the new profile produces a near-normal return, which is 7 to 10 $\mathrm{dB}$ less than that produced by the triangular profile. Far from the normal, the performance deteriorates as with any other shape. This example reveals that there is a scope for optimizing absorber profile shape for reducing reflections.

The depth of the corrugations will have a significant effect on the power reflected from the absorber surface. Fig. 7 shows variation of the reflection coefficient for normal incidence as a functions of height for the triangular profile. The half apex-angle of the triangle is fixed at $14^{\circ}$. Although the reflection coefficient does not drop monotonously, it gener-

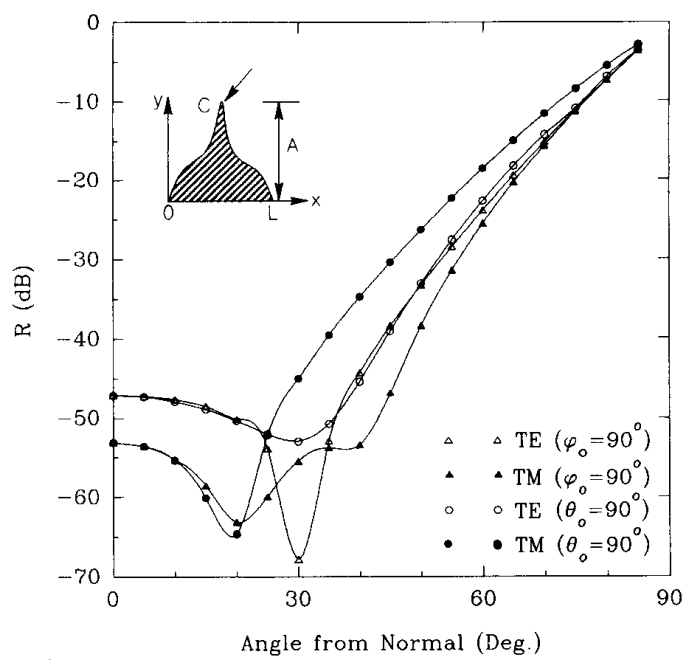

Fig. 6. Variation of reflection coefficient with incidence angle for a variant of triangular profile. The profile is symmetric and the portion $\mathrm{OC}$ is defined as $f(x)=0.5 A[1+\tan \pi \gamma / 2(4 x / L-1) / \tan \pi \gamma / 2]$ with $A / \lambda_{0}=$ $1.016, L / \lambda_{0}=0.508, \gamma=0.61$ and $\epsilon_{r}=1.45-j 0.4$. For $\gamma=0$, the profile reduces to the familiar triangular shape.

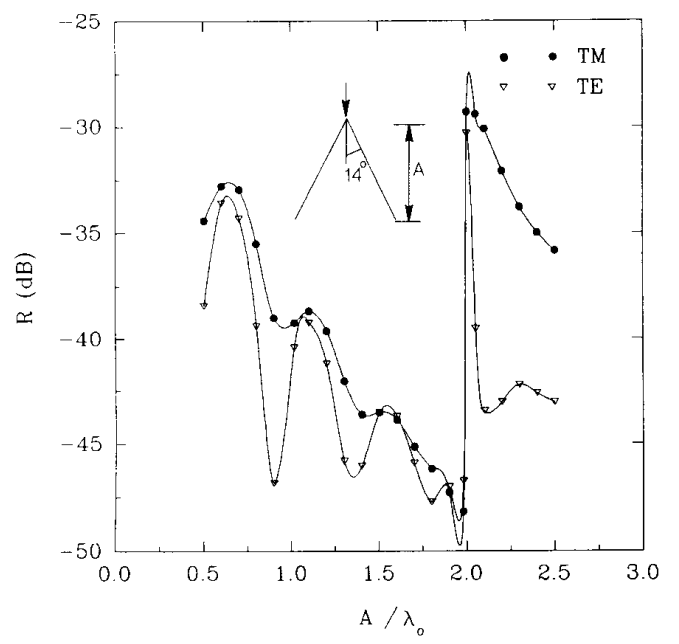

Fig. 7. Variation of reflection coefficient with depth for triangular profile with $\theta_{0}=\phi_{0}=90^{\circ}$ and $\epsilon_{r}=1.45-j 0.4$.

ally decays with increasing absorber depths until the period reaches a wavelength or higher. For the chosen parameters, this happens when $A / \lambda_{0} \approx 2$. For periods less than a freespace wavelength, reflections occur only in the specular direction (the $m=0$ term in (17) and (18)). The next higher order terms $(m= \pm 1)$ just graze the surface when the period is one wavelength and enter the upper half-space for periods greater than one wavelength. Although the specularly reflected term decays with increasing depths, introduction of the higher order terms causes the reflection coefficient to increase rapidly at $A / \lambda_{0} \approx 2$ before it begins to decay. This behaviour is in contrast to widespread belief that the absorber 
performance improves with increasing depths. This is true if one considers only the specular term. For nondispersive materials, Fig. 7 can also be thought of as a variation of reflection coefficient with frequency for fixed dimensions.

Fig. 8 shows the variation of the reflection coefficient for normal incidence as a function of apex angle for the triangular profile. The height of the triangle is held fixed at $A=$ $1.016 \lambda_{0}$ and the half-angle $\alpha$ is varied from $2.5^{\circ}-45^{\circ}$. The reflection coefficient is seen to be approximately steady for values of $\alpha$ up to around $25^{\circ}$ at which time the $m= \pm 1$ terms are excited. At $\alpha \approx 43^{\circ}$, the $m= \pm 2$ terms are excited, and so on. In the limiting case of $\alpha=90^{\circ}$, the reflection coefficient reaches the flat surface value of -18.27 dB.

Finally, we study the variation of reflection coefficient with respect to the loss tangent of the absorbing material. The dielectric constant of the material is assumed to be of the form $\epsilon_{r}=1.45-j \epsilon_{r}^{\prime \prime}$. Loss tangent can be controlled in practice by varying the carbon concentration. Fig. 9 shows the variation of reflection coefficient with $\epsilon_{r}^{\prime \prime}$. The skin depth in the material normalized to the height of the triangle is also plotted along side. It is seen that for both TE and TM polarization, the reflection coefficient reaches a minimum around $\epsilon_{r}^{\prime \prime}=0.18$. Higher values of $\epsilon_{r}^{\prime \prime}$ affect the gradual transition from free-space to the lossy medium. The low reflection coefficient seen in Fig. 9 for zero $\epsilon_{r}^{\prime \prime}$ is slightly misleading. In an actual absorber, the thickness of the panel is finite and not infinitely large as assumed here. Furthermore, the panel is often backed by metal for the purpose of shielding. If $\epsilon_{r}^{\prime \prime}=0$, a low reflection loss for the infinite geometry means that the incident wave is effectively transmitted into the second medium and not absorbed by it. For the more practical geometry this would lead to total reflection by the metal backing! A certain amount of loss must always be present to make it an effective absorber. A higher loss tangent does not necessarily mean higher absorption. For the chosen parameter, the loss tangent must be adjusted such that the skin depth in the lossy medium is approximately equal to the corrugation height in order to make it most effective. More work needs to be done to generalize this remark to arbitrary shapes.

\section{SUMMARY}

Müller type integral equations are formulated to study plane wave scattering from singly periodic, lossy half-space. The material is assumed to be homogeneous, characterized by complex values of permittivity and permeability. The integral equations are solved by the boundary element method with constant elements. Other speed-up techniques are utilized to result in a numerically efficient solution. Comparison is made for special cases to verify the formulation. Arbitrary surface are accomodated by approximating the actual profile by means of linear segments. Power reflection coefficient is calculated for common surface shapes such as rectangular, sinusoidal, triangular, and a variant of triangular. For the specific case of the triangular profile, a systematic study is carried out for the dependence of reflection coefficient on various parameters.

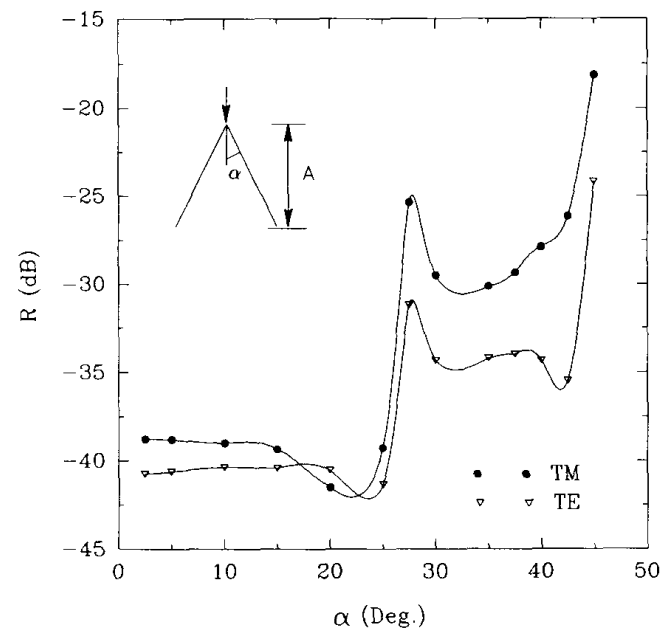

Fig. 8. Variation of reflection coefficient with $\alpha$ for triangular profile with $\theta_{0}=\phi_{0}=90^{\circ}, A / \lambda_{0}=1.016$ and $\epsilon_{r}=1.45-j 0.4$.

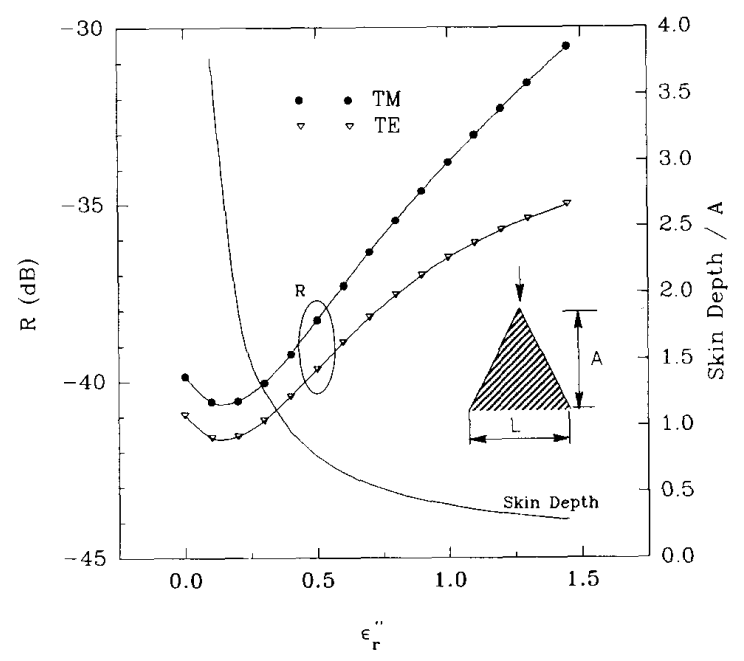

Fig. 9. Variation of reflection coefficient with the imaginary part of $\epsilon_{r}=1.45-j \epsilon_{r}^{\prime \prime}$. The parameters of the triangular profile are $A=1.016 \lambda_{0}$ and $L=0.508 \lambda_{0}$, and the incident wave is normal with $\theta_{0}=\phi_{0}=90^{\circ}$.

A new profile shape is presented that results in lower near-normal reflection coefficient compared to the commonly used triangular shape. For the triangular shape, the reflection coefficient falls off generally with increasing corrugation depths. However, higher return is produced if the period of the corrugations exceeds one free-space wavelength. Low return is produced for apex angles resulting in periods less than one free-space wavelength. Finally, a loss tangent that makes the corrugation depths approximately equal to the skin depth resulted in a low reflection coefficient for the chosen parameters.

\section{REFERENCES}

[1] W. H. Emerson, "Electromagnetic wave absorbers and anechoic chambers through the years," IEEE Trans. Antennas Propagat., vol. AP-21, pp. 484-490, July 1973. 
[2] O. M. Bucci and G. Franceschetti, "Scattering from wedge shaped absorbers," IEEE Trans. Antennas Propagat., vol. AP-19, pp. $96-104$, Jan. 1971

[3] B. T. DeWitt and W. D. Burnside, "Electromagnetic scattering by pyramidal and wedge absorbers," IEEE Trans. Antennas Propa gat., vol. 36, pp. 971-984, July 1988 .

[4] B. T. DeWitt, "Analysis and measurement of electromagnetic scattering by pyramidal and wedge absorbers," Ph.D. dissertation, Dept. Elec. Eng., Ohio State Univ., Columbus, OH, 1986.

[5] C. Cheon and V. V. Liepa, "Full wave analysis of infinitely periodic lossy wedges,"' Symposium Digest, IEEE AP-S Int. Symp., Dallas, TX, pp. 618-621, June 1990

[6] R. Petit, Ed., Electromagnetic Theory of Gratings. New York: Springer-Verlag, 1980.

[7] N. Morita, "Surface integral representations for electromagnetic scattering from dielectric cylinders," IEEE Trans. Antennas Propagat. vol. AP-26, pp. 261-266, Mar. 1978.

[8] A. A. Kishk and L. Shafai, "Different formulations for numerical solutions of single or multibodies of revolution with mixed boundary conditions," IEEE Trans. Antennas Propagat., vol. AP-34, pp. 666-673, May 1986.

[9] C. Müller, Foundations of the Mathematical Theory of Electromagnetic Waves. New York: Springer-Verlag, 1969, ch. 6, pp. 297-320.

[10] J. R. Wait, "Scattering of a plane wave from a circular dielectric cylinder at oblique incidence," Canad. J. Phys., vol. 33, pp. $189-195,1955$.

[11] K. A. Zaki and A. R. Neureuther, "Scattering from a perfectly conducting surface with a sinusoidal height profile: TE polarization," IEEE Trans. Antennas Propagat., vol. AP-19, pp. 208-214, Mar. 1971.

[12] T. C. H. Tong and T. B. A. Senior, "Scattering of electromagnetic waves by a periodic surface with arbitrary profile," Dept. Elec. Comput. Eng., Radiation Lab., Univ. Michigan, Ann Arbor, MI, Sci. Rep. 13, AFCRL-72-0258, 1972.

[13] C. A. Brebbia and S. Walker, Boundary Element Techniques in Engineering. Boston, MA: Butterworth, 1980.

[14] K. Yashiro and S. Ohkawa, "Boundary element method for electromagnetic scattering from cylinders,'” IEEE Trans. Antennas Propagat., vol. AP-33, pp. 383-389, Apr. 1985.
[15] S. Singh, W. F. Richards, J. R. Zinecker and D. R. Wilton, "Accelerating the convergence of series representing the free space periodic Green's function," IEEE Trans. Antennas Propagat., vol. 38, pp. 1958-1962, Dec. 1990

[16] D. E. Terman and K. K. Mei, "Application of the unimoment method to scattering from periodic dielectric structures," $J$. Opt. Soc. Amer, vol. 68, no. 6, pp. 775-783, June 1978 .

[17] Y. Nakata and M. Koshiba, "Boundary-element analysis of planewave diffraction from groove type dielectric and metallic gratings," J. Opt. Soc. Amer., ser. 2, vol. 7, no. 8, pp. 1494-1502, Aug 1990.

[18] E. Marx, "Computer fields near the edge of a dielectric wedge," IEEE Trans. Antennas Propagat., vol. 38, pp. 1438-1442, Sept. 1990.

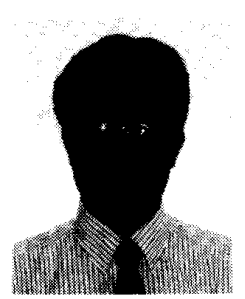

Ramakrishna Janaswamy (S'82-S'83-M'83$M^{\prime} 86$ ) received the $B$. Tech degree (with Gold Medal) in electronics and communication engineering from R.E.C. Warangal, India, in 1981, the $M . T e c h$ degree in microwave and radar engineering from I.I.T., Kharagpur, India, in 1983, and the Ph.D. degree in electrical engineering from the University of Massachusetts, Amherst, in 1986.

From August 1986 to May 1987, he was an Assistant Professor of electrical engineering at Wilkes University, Wilkes-Barre, PA. During the summer of 1987, he was a Visiting Assistant Professor in the Department of Electrical and Computer Engineering at the University of Illinois, Urbana. In September 1987, he joined the Department of Electrical and Computer Engineering at the Naval Postgraduate School, Monterey, CA, where is currently an Associate Professor. His current research interests are in the areas of electromagnetic scattering, wide-band antennas, and propagation over irregular terrain.

Dr. Janaswamy is a member of the Commission B of the International Union of Radio Science and the Electromagnetics Academy. 